International Journal of Pure and Applied Mathematics

Volume 85 No. 4 2013, 635-651

ISSN: 1311-8080 (printed version); ISSN: 1314-3395 (on-line version)

url: http://www.ijpam.eu

doi: http://dx.doi.org/10.12732/ijpam.v85i4.2

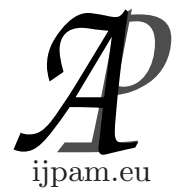

\title{
ENUMERATION TECHNIQUE FOR SOLVING \\ LINEAR FUZZY SET COVERING PROBLEM
}

\author{
Ratnesh Rajan Saxena ${ }^{1}$, Rashmi Gupta $^{2} \S$ \\ ${ }^{1}$ Department of Mathematics \\ Deen Dayal Upadhyaya College \\ University of Delhi \\ Delhi, 110015, INDIA \\ ${ }^{2}$ Department of Mathematics \\ University of Delhi \\ Delhi, 110007, INDIA
}

\begin{abstract}
In this paper a method for solving linear fuzzy set covering problem is defined. Set covering problems belong to the class of 0-1 integer programming problems that are NP-complete. Many applications arises having the set covering problems, switching theory, testing of VLSI circuits and line balancing often take on a set covering structure. Linear set covering problems involving coefficients in the objective function with some lack of precision are usual. To solve them several approaches have been proposed. In this paper a solution algorithm to fuzzy linear set covering problem is suggested. In order to defuzzify the problem the concept of vector ranking function is given and for obtaining efficient solution to the problem, an algorithm is constructed with a weighted sum approach.An example is illustrated to demonstrate the correctness of the proposed solution algorithm.
\end{abstract}

Key Words: set covering, fuzzy number, vector ranking function

Received: December 26, 2011

(C) 2013 Academic Publications, Ltd. url: www.acadpubl.eu

${ }^{\S}$ Correspondence author 


\section{Introduction}

The set covering problem is one of the most famous problems in complexity and approximation theory. Given a set $\mathcal{C}=\left\{c_{1}, \ldots, c_{n}\right\}$ of elements and a collection $\mathcal{S}=\left\{S_{1}, \ldots, S_{n}\right\}$ of subsets of $\mathcal{C}$, the goal is to find a subset $S^{\prime} \subset S$ of minimum cardinality such that $\bigcup_{S_{\mathrm{j}} \in S^{\prime}} S_{j}=\mathcal{C}$.In the weighted set covering problem, a weight $w_{i}$ being given for every set $S_{i}$, we want to minimize $\sum_{S_{\mathrm{i}} \in S^{\prime}} w_{i}$, or, equivalentely, $\sum_{i=1}^{m} w_{i} s_{i}$ where $S_{i}=1$, if $S_{i}$ is chosen in the solution and $S_{i}=0$ otherwise.

Often, a decision maker is supposed to be able to exactly fit all numerical values taking part in the problem, but frequently he does not know those exact values, rather he knows some approximations of them and therefore he may feel more comfortable in specifying vague(fuzzy) values rather than point values. This paper considered the case in which a decision maker has a set covering problem in which the coefficients defining the objective function are given by fuzzy numbers whereas the constraints set is a conventional one.Thus the existence of the membership function is assumed.These membership functions will capture the knowledge that the decision maker has about each cost taking part into account.

The fuzzy set theory has been applied in many disciplines such as operations research, managemental sciences, control theory, artificial intelligence etc. The growth of applications of the fuzzy set theory has been accumulating. At the turn of the century, reducing complex real world system into precise mathematical model is the main trend in science and engineering. In the middle of century, operations research began to be applied to real-world decision-making problems and this became one of the most important fields in science and engineering. Unfortunately, real world situations are often not so deterministic. Thus precise mathematical models are not enough to tackle all practical problems. In this paper the concept of optimality for linear fuzzy set covering problems with fuzzy parameters is obtained.

The paper has the following structure. In Section 2, linear set covering problems, fuzzy linear set covering problems and some definitions are presented. In Section 3, a vector ranking function is presented to convert linear fuzzy set covering problem to a multiobjective linear set covering problem. In Section 4, some basic concepts of optimality for multiobjective linear integer programming problems are given. In Section 5, a weigted sum approach is developed to solve fuzzy linear set covering problem. In Section 6, numerical example is being 
given in support of the algorithm. Section 7, concludes the paper.

\section{Theoretical Development}

\subsection{Linear set covering Problems (CP)}

Set covering problem is a classical problem in computer science and complexity theory.Set covering problem is one of most important discrete optimization problem because it serves as a model for real world problems. Real world problem that can be modeled as set covering problem include facility location problem, airline crew scheduling, nurse scheduling problem, etc.Consider a set $I=$ $\{1,2, \ldots, m\}$ and a set $P=\left\{P_{1}, P_{2}, \ldots, P_{n}\right\}$ where $P_{j} \subseteq I, j \in J=[1,2, \ldots, n]$. A subset $J^{*}$ of $J$ is said to be a cover of $I$ if $\bigcup_{j \in J^{*}} P_{j}=I$. Let a cost $c_{j}>0$ be associated with every $j \in J$. The total cost of the cover $J^{*}$ is equal to $\sum_{j \in J^{*}} c_{j}$. The linear set covering problem $(\mathrm{CP})$ is to find a cover of minimum cost subject to the condition that atleast one of the utility is satisfied and objective function is linear function. Mathematically, the problem is

$$
\begin{aligned}
& \text { (CP) } \min z=\sum_{j=1}^{n} c_{j} x_{j} \\
& \text { subject to } \sum_{j=1}^{n} a_{j} x_{j} \geq 1, \quad i \in 1 \\
& \quad x_{j}=0 \text { or } 1, \quad j \in J .
\end{aligned}
$$

where

$$
x_{j}=\left\{\begin{array}{ll}
1 & \text { if } j \text { is in the cover } \\
0 & \text { otherwise }
\end{array} \text { and } a_{i j}=\left\{\begin{array}{ll}
1 & \text { if } i \in P_{j} \\
0 & \text { otherwise }
\end{array} .\right.\right.
$$

It is assumed that $c_{j}$ 's are non-negative.

In matrix form, $(\mathrm{CP})$ can be written as

$$
\begin{aligned}
& \min z=C x \\
& \text { subject to } A x \geq b
\end{aligned}
$$

where $x^{T}=\left(x_{1}, x_{2}, \ldots, x_{n}\right)$ with $x_{j}=0$ or $1, j=1,2, \ldots, n$. Here $C=$ $\left(c_{1}, c_{2}, \ldots, c_{n}\right) \in R^{n}$ is a row vectors. $A$ is an $m \times n$ matrix of zeros and ones and $b^{T}=(1,1, \ldots, 1)$ is a row vector of ones. 


\section{Definitions.}

2.1. Cover Solution: A solution $X$ which satisfies (CP) (2.1) is said to be a cover solution.

2.2. Redundant Cover: For any cover $J$, a column $j^{*} \in J$ is said to be redundant if $J-\left\{j^{*}\right\}$ is also a cover.

If a cover contains one or more redundant columns, it is called a redundant cover. Column $j^{*}$ is redundant with respect to the cover $J$ iff $\sum_{j \in J} a_{i j} \geq 2$ for all $i \in P_{j}$.

2.3. Prime Cover: A cover $j^{*}$ is said to be a prime cover, if none of the columns corresponding to $j \in j^{*}$ is redundant. A solution corresponding to the prime cover is called a prime cover solution.

2.4. Fuzzy Number: A fuzzy set $\widetilde{A}$ on $\mathbb{R}$ is called a fuzzy number if it satisfies the following two conditions:

(i) It's membership function is upper semi continuous.

(ii) There exists three intervals $[a, b],[b, c]$ and $[c, d]$ such that membership function of $\widetilde{A}$ is increasing on $[a, b]$, equal to 1 on $[b, c]$, decreasing on $[c, d]$ and equal to 0 anywhere else.

\subsection{Fuzzy Linear Set Covering Problem (FCP)}

If the coefficient in the objective function of problem (CP) (2.1) becomes fuzzy in nature then problem (CP) (2.1) translates to fuzzy set covering problem (FCP) i.e.

$$
\begin{aligned}
& \text { (FCP) } \min z=\sum_{j=1}^{n} \widetilde{c}_{j} x_{j} \\
& \text { subject to } \sum_{j=1}^{n} a_{i j} x_{j} \geq 1, \quad i \in I \\
& x_{j}=0 \text { or } 1, \quad j \in J
\end{aligned}
$$

where

$$
x_{j}=\left\{\begin{array}{ll}
1 & \text { if } j \text { is in the cover } \\
0 & \text { otherwise }
\end{array} \text { and } a_{i j}=\left\{\begin{array}{ll}
1 & \text { if } i \in P_{j} \\
0 & \text { otherwise }
\end{array} .\right.\right.
$$


Let $x=\left(x_{1}, x_{2}, \ldots, x_{n}\right)^{T}, y=\left(y_{1}, y_{2}, \ldots, y_{n}\right)^{T} \in R^{n}$ be two vectors. Then $x \leq y$ iff $x_{i} \leq y_{i}$ for all i belong to $N=\{1,2, \ldots, n\} ; x<y$ iff $x_{i} \leq y_{i}$ for all $i \in N ; x \neq y$ iff $x_{i} \neq y_{i}$ for some $i \in N$.

Notation. $F(\mathbb{R})$ denotes the set of all fuzzy numbers.

\section{Ordering Elements of $F(\mathbb{R})$}

In order to compare the fuzzy numbers, define the ordering of fuzzy numbers by mapping each fuzzy number into a point of the real line, where a natural order already exists.

Remark. More than one ranking function can be defined (Bortolan and Degani, 1985; Cadenas and Verdegay, 1997).

Based on the decision maker's preferences, assume there exists $k$ important attributes associated to fuzzy number $\widetilde{A}$ such that $i$ th of them can be characterized by the ranking function $R_{i}: F(\mathbb{R}) \rightarrow R$. In this case, consider a crisp $k$-dimensional vector, $R(\widetilde{A})$, to $\widetilde{A}$ as follows:

$$
R(\widetilde{A})=\left(R_{1}(\widetilde{A}), R_{2}(\widetilde{A}), \ldots, R_{k}(\widetilde{A})\right)^{T} .
$$

\section{Definitions.}

3.1. Vector Ranking Function The vector function $R($.$) , defined as$ above, is called a vector ranking function. Moreover let $\widetilde{A}$ and $\widetilde{B}$ belong to $F(\mathbb{R})$, then:

$$
\begin{array}{ccc}
\widetilde{A} \leq \underset{R}{ } \widetilde{B} & \text { iff } & R(\widetilde{A}) \leq R(\widetilde{B}) \\
\widetilde{A} \underset{R}{\widetilde{B}} & \text { iff } & R(\widetilde{A})<R(\widetilde{B}) \\
\widetilde{A} \underset{R}{\widetilde{B}} \widetilde{B} & \text { iff } & R(\widetilde{A}) \neq R(\widetilde{B}) \\
\widetilde{A} \neq \widetilde{B} & \text { iff } & R(\widetilde{A}) \neq R(\widetilde{B}) \\
R & &
\end{array}
$$

Similarly

$$
\widetilde{A} \underset{R}{\geq} \widetilde{B} \quad \text { iff } \quad \widetilde{B} \underset{R}{ } \widetilde{A} \quad \text { and } \quad \widetilde{A}>\widetilde{B} \quad \text { iff } \quad \widetilde{B} \underset{R}{<} \widetilde{A}
$$

Vector Ranking Functions for $k=1,2,3$ 
(i) Let $\widetilde{A}$ be a fuzzy number. Roubens ranking function is a vector ranking function with $k=1$ when the decision maker choose $R_{1}($.$) as the only$ characteristic for ordering. It is defined as:

$$
R(\widetilde{A})=\frac{1}{2} \int_{0}^{1}\left(\inf \widetilde{A}_{r}+\sup \widetilde{A}_{r}\right) d r
$$

where $\widetilde{A}_{r}$ is an $r$-cut of $\widetilde{A}$ i.e. $\widetilde{A}_{r}=\{x \in R \mid \widetilde{A}(X) \geq r\}, 0<r \leq 1$.

(ii) For $k=2, R(\widetilde{A})=(E(\widetilde{A}),-\operatorname{var}(\widetilde{A}))^{T}$ where $E(\widetilde{A})$, and $\operatorname{var}(\widetilde{A})$ are the expectation and variance of the density function associated with $(\widetilde{A})$ (Delgado et al., 1998a).

(iii) For $k=3, R(\widetilde{A})=(V(\widetilde{A}), A(\widetilde{A}), F(\widetilde{A}))^{T}$ where $V(\widetilde{A}), A(\widetilde{A})$ and $F(\widetilde{A})$ are value, ambiguity and fuzziness of $\widetilde{A}$, respectively, which are defined as:

$$
\begin{aligned}
& V(\widetilde{A})=\int_{0}^{1} r\left[L_{\widetilde{A}}(r)+R_{\widetilde{A}}(r)\right] d r \\
& A(\widetilde{A})=\int_{0}^{1} r\left[R_{\widetilde{A}}(r)-L_{\widetilde{A}}(r)\right] d r \\
& F(\widetilde{A})=\int_{0}^{1 / 2}\left[R_{\widetilde{A}}(r)-L_{\widetilde{A}}(r)\right] d r+\int_{1 / 2}^{1}\left[L_{\widetilde{A}}(r)-R_{\widetilde{A}}(r)\right] d r
\end{aligned}
$$

where both $L_{\widetilde{A}}($.$) and R_{\widetilde{A}}($.$) are from [0,1]$ to $\mathbb{R}$ and defined as:

$$
\begin{aligned}
& L_{\widetilde{A}}(r)= \begin{cases}\inf \left\{x \mid x \in \widetilde{A}_{r}\right\} & \text { if } r \in(0,1] \\
\inf \{x \mid x \in \operatorname{Supp}(\widetilde{A})\} & \text { if } r=0\end{cases} \\
& R_{\widetilde{A}}(r)= \begin{cases}\sup \left\{x \mid x \in \widetilde{A}_{r}\right\} & \text { if } r \in(0,1] \\
\sup \{x \mid x \in \operatorname{Supp}(\widetilde{A})\} & \text { if } r=0\end{cases}
\end{aligned}
$$

and

$$
\operatorname{Supp}(\widetilde{A})=\{x \in R \mid \widetilde{A}(x)>0\} .
$$




\section{Multiobjective Linear Integer Programming Problem}

In this section the basis of multiobjective linear integer programming problem have been reviewed.

Consider the model:

$$
\begin{aligned}
(\mathrm{MIP}) \quad \min z(x)=C x \\
\text { subject to } A x \geq b \\
x=0 \text { or } 1
\end{aligned}
$$

where $C$ is a $k \times n$ matrix of coefficients of the linear objective function, $A \in R^{m \times n}$ and $b \in R^{m}$ and $x \in R^{n}$, is called a multiobjective linear integer programming problem (MIP).

Let $X=\left\{x \in R^{n} \mid A x \geq b, x=0\right.$ or 1$\}$ be the feasible region of problem (MIP).

\section{Definitions.}

4.1. Complete Optimal Solution: A point $x^{*} \in X$ is called a Complete optimal solution for (MIP) iff $z\left(x^{*}\right) \leq z(x) \forall x \in X$.

4.2. Pareto Optimal Solution: A point $x^{*} \in X$ is called a Pareto optimal solution for (MIP) iff there does not exist another $x \in X$ such that $z(x) \leq z\left(x^{*}\right)$ and $z(x) \neq z\left(x^{*}\right)$.

4.3. Weak Pareto Optimal Solution: A point $x^{*} \in X$ is called a Weak pareto optimal solution for (MIP) iff there does not exists another $x \in X$ such that $z(x)<z\left(x^{*}\right)$.

Let $E^{c}, E^{p}$ and $E^{w p}$ denote the set of all complete optimal solution, pareto optimal and weak pareto optimal solutions for MIP respectively, then $E^{c} \subseteq$ $E^{p} \subseteq E^{w p}$.

\section{Development of Algorithm to Solve Fuzzy Linear Set Covering Problem}

In this section a method is investigated to solve a linear set covering problem with fuzzy parameters. Moreover, suppose that $R$ is a given vector ranking function 
(FCP) Fuzzy linear set covering problem:

$$
\begin{aligned}
& \min \widetilde{z}=\frac{\bar{R}}{c} x \\
& \text { subject to } x \in X
\end{aligned}
$$

where $\widetilde{c}=\left(\widetilde{c}_{1}, \widetilde{c}_{2}, \ldots, \widetilde{c}_{n}\right) \in(F(R))^{n}$ is called a multiobjective fuzzy set covering problem.

\section{Definitions.}

5.1. $R$-optimal solution: A point $x^{*} \in X$ is called an $R$-optimal solution for (FCP) (5.1) iff $\widetilde{c} x^{*} \underset{R}{\operatorname{co} x} \forall x \in X$.

5.2. $R$-efficient solution: A point $x^{*} \in X$ is called an $R$-efficient solution for (FCP) (5.1) iff there does not exist another $x \in X$ such that $\widetilde{c} x \leq \underset{R}{c} x^{*}$ and $\widetilde{c} x \neq \underset{R}{*} \widetilde{c} x^{*}$.

5.3. $R$-weak efficient solution: A point $x^{*} \in X$ is called an $R$-weak efficient solution for (FCP) (5.1) iff there does not exist another $x \in X$ such that $\widetilde{c} x \underset{R}{<} \widetilde{c} x^{*}$.

Denote $R$-optimal, $R$-efficient and $R$-weak efficient solution sets by $X^{\text {ro }}$, $X^{r e}, X^{r w}$ respectively, then $X^{r o} \subseteq X^{r e} \subseteq X^{r w}$.

Now associated with the model (FCP) (5.1), consider the following (MOCP) problem.

$$
\begin{aligned}
& \min \{z(x)=R(\widetilde{c} x) \mid x \in X\} \\
& \text { where } R(.)=\left(R_{1}(.), R_{2}(.), \ldots, R_{k}(.)\right\}
\end{aligned}
$$

Following theorems provides the relationship between the optimal solution of the (FCP) (5.2) and (MOCP) (5.1).

Theorem 5.1. A point $x^{*} \in X$ is an $R$-optimal solution for the $(F C P)(5.1)$ iff $x^{*}$ is a complete optimal solution or $(M O C P)(5.2)$.

Proof. $(\Rightarrow)$ Assume that $x^{*} \in X$ is an $R$-optimal solution of the (FCP) (5.1), then for any $x \in X, \widetilde{c} x^{*} \leq \frac{\leq}{R} \widetilde{c} x$. Now by definition of $\frac{\leq}{R}, R\left(\widetilde{c} x^{*}\right) \leq R(\widetilde{c} x)$, for all $x \in X$, and hence $z\left(x^{*}\right) \leq z(x) \forall x \in X$.

Therefore, $x^{*}$ is a complete optimal solution for the (MOCP) (5.2).

$(\Leftarrow)$ Assume $x^{*}$ is a complete optimal solution for the (MOCP) (5.2), then for all $x \in X, z\left(x^{*}\right) \leq z(x)$, and hence $R\left(\widetilde{c} x^{*}\right) \leq R(\widetilde{c} x)$. Therefore $\widetilde{c} x^{*} \leq \widetilde{c} x$ for all $x \in X$. This implies $x^{*}$ is an $R$-optimal solution for the (FCP) (5.1). 
Theorem 5.2. A point $x^{*} \in X$ is an $R$-efficient solution for $(F C P)(5.1)$ iff $x^{*}$ is a pareto optimal solution for (MOCP) (5.2).

Proof. $(\Rightarrow)$ Let $x^{*} \in X$ be an $R$-efficient solution for (FCP) (5.1). On the contrary, suppose that $x^{*}$ is not a pareto optimal solution for (MOCP) (5.2), i.e., there exists $\bar{x} \in X$ such that $z(\bar{x}) \leq z\left(x^{*}\right)$ and $z(\bar{x}) \neq z\left(x^{*}\right)$, hence $R(\widetilde{c} \bar{x}) \leq R\left(\widetilde{c} x^{*}\right)$ and $R(\widetilde{c} \bar{x}) \neq R\left(\widetilde{c} x^{*}\right)$. This implies $\widetilde{c} \bar{x} \leq_{R} \widetilde{c} x^{*}$ and $\widetilde{c} \bar{x} \neq \widetilde{c} x^{*}$ which contradicts that $x^{*} \in X$ is an efficient solution for the (FCP) (5.1).

$(\Leftarrow)$ Let $x^{*} \in X$ be a pareto optimal solution for (MOCP) (5.2). On the contrary, suppose that $x^{*} \in X$ is not an $R$-efficient solution for the (FCP) (5.1), i.e. there exists an $\bar{x} \in X$ such that $\underset{c}{\bar{c}} \underset{R}{\leq} \underset{c}{c} x^{*}$ and $\underset{c}{\tilde{c} \bar{x}} \underset{R}{\neq} \widetilde{c} x^{*}$ this implies and $R(\widetilde{c} \bar{x}) \leq R\left(\widetilde{c} x^{*}\right)$ and $R(\widetilde{c} \bar{x}) \neq R\left(\widetilde{c} x^{*}\right)$.

This means $z(\bar{x}) \geq z\left(x^{*}\right)$ and $z(\bar{x}) \neq z\left(x^{*}\right)$ which contradicts that $x^{*} \in X$ is a pareto optimal solution for (MOCP) (5.2).

Theorem 5.3. A point $x^{*} \in X$ is an $R$-weak efficient solution for $(F C P)(5.1)$ iff $x^{*}$ is a weak pareto optimal solution for $(M O C P)(5.2)$.

Proof. $(\Rightarrow)$ Let $x^{*} \in X$ be a $R$-weak efficient solution for (FCP) (5.1). On the contrary suppose that $x^{*}$ is not a weak pareto optimal solution for (MOCP) (5.2) this implies there exist another $x \in X$ such that $z(x)<z\left(x^{*}\right)$ hence $R(\widetilde{c} x)<R\left(\widetilde{c} x^{*}\right)$ this implies $\widetilde{c} x \underset{R}{<} \widetilde{c} x^{*}$ which contradicts that $x^{*} \in X$ is an $R$-weak efficient solution for (FCP) (5.1).

$(\Leftarrow)$ On the other hand let $x^{*} \in X$ be a weak pareto optimal solution for (MOCP) (5.2). On the contrary, we suppose that $x^{*} \in X$ is not an $R$-weak efficient solution for the (FCP) (5.1) i.e. there exists an $\bar{x} \in X$ such that $\widetilde{c} \bar{x}<$ $\widetilde{c} x^{*}$ this implies $R(\widetilde{c} \bar{x})<R\left(\widetilde{c} x^{*}\right)$. This means $z(\bar{x})<z\left(x^{*}\right)$ which contradicts that $x^{*} \in X$ is a weak pareto optimal solution for (MOCP) (5.2).

\section{Weighted Sum Approach}

Now to generate pareto (weak pareto) optimal solution of (FCP) (5.2) the weighted sums of objective functions approach is being used, i.e., consider the solution of the following weighted problem:

$$
\text { (WP) } \min \{w R(\tilde{c} x) \mid x \in X\}
$$


where $w \in W=\left\{w \in \mathbb{R} \mid w \geq 0, \sum_{j=1}^{k} w_{j}=1\right\}$ for finding R-efficient solution or R-weak efficient solution of the (MOCP) (5.2), it suffices to use the following theorems:

Theorem 6.1. Let a point $x^{*} \in X$ be an optimal solution of weighted problem (WP) (5.3) for some $w>0$, then $x^{*}$ is an R-efficient solution for (FCP) (5.1).

Proof. Let $x^{*} \in X$ be an optimal solution of weighted problem (WP) (5.3) for some $w>0$ and let if possible $x^{*}$ be not an R-efficient solution of (FCP) (5.1). From Theorem 5.2 it follows that $x^{*}$ is not a pareto optimal solution of(MOCP) (5.2). Therefore there exists an $\bar{x} \in X$ such that $z(\bar{x}) \leq z\left(x^{*}\right)$ and $z(x) \neq z\left(x^{*}\right)$. Hence $R(\tilde{c} \bar{x}) \leq R\left(\tilde{c} x^{*}\right)$ and $R(\widetilde{c} \bar{x}) \neq R\left(\widetilde{c} x^{*}\right)$. This means $R_{j}(\widetilde{c} \bar{x}) \leq R_{j}\left(\widetilde{c} x^{*}\right)$ for all $j=\{1,2, \ldots, k\}$ and there exist atleast a $j_{0} \in\{1,2, \ldots, k\}$ such that $R_{j_{0}}(\widetilde{c} \bar{x})<R_{j_{0}}\left(\widetilde{c} x^{*}\right)$, now since $w>0$, therefore

$$
w R\left(\widetilde{c} x^{*}\right)=\sum_{j=1}^{k} w_{j} R_{j}\left(\widetilde{c} x^{*}\right)<\sum_{j=1}^{k} w_{j} R_{j}(\widetilde{c} \bar{x})=w R(\widetilde{c} \bar{x})
$$

This contradicts that $x^{*}$ is an optimal solution of weighted problem (WP) (5.3).

Lemma 1. Let $\bar{x} \in S$ and $D$ be an $n \times n$ diagonal matrix with

$$
d_{j j}= \begin{cases}-1 & \text { if } \bar{x}_{j}=0 \\ 0 & \text { otherwise }\end{cases}
$$

where $S=\left\{x \in R^{n} \mid A x=b\right\}$.

Then, $\bar{x} \in E^{p} \Leftrightarrow$ the system $C u \leq 0, C u \neq 0, D u \leq 0, A u=0$ has no solution $u \in \mathbb{R}^{n}$.

Proof. $(\Rightarrow)$ First let $\bar{x} \in E^{p}$ and suppose $u$ satisfies the system. Let $\widehat{x}=$ $\bar{x}+\alpha u$. Then, there exists an $\bar{\alpha}>0$ such that for all $\alpha \in[0, \bar{\alpha}] ., \widehat{x} \in S$. But $c \widehat{x}-c \bar{x}=\alpha c u \leq 0$ and $\alpha c u \neq 0$. This implies that $\bar{x} \notin E^{p}$ which is a contradiction. Hence the system is not solvable.

$(\Leftarrow)$ Suppose the system is not solvable, to prove that $\bar{x} \in E^{p}$. Let $x \in S$ and let $u=x-\bar{x}$. Consequently $A u=0$ and $D u \leq 0$. Hence it is not true that $C u \leq 0 . C u \neq 0$. Thus it is not true that $C x \leq C \bar{x}, C x \neq C \bar{x}$. Since $x \in S$ was arbitrary, $\bar{x}$ is pareto optimal solution. 
Lemma 2. Let $\bar{x} \in S$ and $D$ be defined as in Lemma 1. Then, $\bar{x} \in E^{p}$ iff there exist $\pi \in R^{k}, y^{3} \in R^{n}$ and $y^{4} \in R^{m}$ such that

$$
\begin{aligned}
& c^{T} \pi+D^{T} y^{3}+A^{T} y^{4}=0 \\
& \pi>0, y^{3} \geq 0
\end{aligned}
$$

Proof. Tucker's theorem of the alternative (Mangasarian 1965) and Lemma 1.

Theorem 6.2. Let a point $x^{*} \in X$ be an R-efficient solution for (FCP) (5.1), then $x^{*}$ is an optimal solution of weighted problem (WP) (5.3) for some $w>0$.

Proof. $(\Rightarrow)$ Since $x^{*} \in E^{p}$ therefore by Lemma 2 there exist $\pi \in R^{k}, y^{3} \in$ $R^{m}$ and $y^{4} \in R^{m}$ such that the system

$$
\begin{aligned}
& c^{T} \pi+D^{T} y^{3}+A^{T} y^{4}=0 \\
& \pi>0, y^{3} \geq 0 \text { is consistent. }
\end{aligned}
$$

Allowing

$$
\alpha=\sum_{i=1}^{k} \pi_{i}>0 .
$$

Rewrite the above system as

$$
\begin{aligned}
& \left.c^{T} \lambda\right) \alpha+D^{T} y^{3}+A^{T} y^{4}=0 \\
& \lambda \in W, y^{3} \geq 0
\end{aligned}
$$

But by Tucker's theorem of the alternative, the system

$$
\left(\lambda^{T} c\right) u \leq 0, \quad\left(\lambda^{T} c\right) u \neq 0, \quad D u \leq 0, \quad A u=0
$$

has no solution. Now for any $x \in S, D\left(x-x^{*}\right) \leq 0$ and $A\left(x-x^{*}\right)=0$. Hence, it is not true that $\lambda^{T} c\left(x-x^{*}\right) \leq 0$ and $\lambda^{T} c\left(x-x^{*}\right) \neq 0$. This implies that $\lambda^{T} c x \geq \lambda^{T} c x^{*}$, since $\lambda^{T} c x^{*}$ is a scalar.

Theorem 6.3. A point $x^{*}$ is an optimal solution for weighted problem (WP) (5.3) for some $w \in W$ then $x^{*}$ is an $R$-weak efficient solution for (FCP) (5.1). 
Proof. Let $x^{*}$ be an optimal solution for weighted problem (WP) (5.3) for some $w \in W$. then by theorem (5.4) $x^{*}$ is an R-efficient solution for (FCP) (5.1). Since $R^{E} \subset R^{W E}$ therefore $x^{*}$ is an R-weak efficient solution for (FCP) (5.1).

Theorem 5.4 and Theorem 5.5 says that, each $x \in X$ which is an optimal solution of the weighted problem (WP) (5.3) for any $w>0$ is an R-efficient optimal solution for fuzzy multiobjective set covering problem (FCP) (5.1). On the other hand at least one $w>0$ is associated with every R-efficient optimal solution, that causes the weighted problem (WP) (5.3), to have it as an optimal solution.

\section{Numerical Examples}

To illustrate the efficiency of the proposed method consider the following examples:

Example 7.1. Consider the problem

$$
\min z(x)=\widetilde{c}_{1} x_{1}+\widetilde{c}_{2} x_{2}+\widetilde{c}_{3} x_{3}
$$

subject to

$$
\begin{aligned}
& x_{1}+x_{2} \geq 1 \\
& x_{2}+x_{3} \geq 1 \\
& x_{1}+x_{3} \geq 1 \\
& x_{1}=0 \text { or } 1, \quad i=1,2,3
\end{aligned}
$$

where the membership functions of $\widetilde{c}_{1}, \widetilde{c}_{2}$ and $\widetilde{c}_{3}$ are

$$
\widetilde{c}_{1}(x)= \begin{cases}0 & x<5 \\ x-5 & 5 \leq x<6 \\ 1 & 6 \leq x \leq 7 \\ (20-x) / 13 & 7<x \leq 20 \\ 0 & 20<x\end{cases}
$$




$$
\begin{gathered}
\widetilde{c}_{2}(x)= \begin{cases}0 & x<16 \\
x-16 & 16 \leq x<17 \\
1 & 17 \leq x \leq 18 \\
(40-x) / 22 & 18<x \leq 40 \\
0 & 40<x\end{cases} \\
\widetilde{c}_{3}(x)= \begin{cases}0 & x<24 \\
x-24 & 24 \leq x<25 \\
1 & 25 \leq x \leq 26 \\
(50-x) / 24 & 26<x \leq 50 \\
0 & 50<x\end{cases}
\end{gathered}
$$

where $V(\widetilde{A}), A(\widetilde{A})$ and $F(\widetilde{A})$ are as defined before: Note that

$$
\begin{array}{lll}
V\left(\widetilde{c}_{1}\right)=8.5, & v\left(\widetilde{c}_{2}\right)=21, & v\left(\widetilde{c}_{3}\right)=88 / 3 \\
A\left(\widetilde{c}_{1}\right)=17 / 6 & A\left(\widetilde{c}_{2}\right)=13 / 3, & A\left(\widetilde{c}_{3}\right)=14 / 3 \\
F\left(\widetilde{c}_{1}\right)=3.5 & F\left(\widetilde{c}_{2}\right)=23 / 4, & F\left(\widetilde{c}_{3}\right)=25 / 4
\end{array}
$$

The following (MOLP) is associated with the problem (6.1):

$$
\begin{aligned}
(\operatorname{MOLP}) \min z(x)= & \left(8.5 x_{1}+21 x_{2}+\frac{88}{3} x_{3}, \frac{17}{6} x_{1}+\frac{13}{3} x_{2}\right. \\
& \left.+\frac{14}{3} x_{3}, 3.5 x_{1}+\frac{23}{4} x_{2}+\frac{25}{4} x_{3}\right)
\end{aligned}
$$

subject to

$$
\begin{aligned}
& x_{1}+x_{2} \geq 1 \\
& x_{2}+x_{3} \geq 1 \\
& x_{1}+x_{3} \geq 1 \\
& x_{i}=0 \text { or } 1 \quad i=1,2,3 .
\end{aligned}
$$

To solve the above problem, the following weighted problem is considered:

$$
\begin{aligned}
\min z(x)= & w_{1}\left(8.5 x_{1}+21 x_{2}+\frac{88}{3} x_{3}\right)+w_{2}\left(\frac{17}{6} x_{1}+\frac{13}{3} x_{2}+\frac{14}{3} x_{3}\right) \\
& +w_{3}\left(3.5 x_{1}+\frac{23}{4} x_{2}+\frac{25}{4} x_{3}\right)
\end{aligned}
$$


subject to

$$
\begin{aligned}
& x_{1}+x_{2} \geq 1 \\
& x_{2}+x_{3} \geq 1 \\
& x_{1}+x_{3} \geq 1 \\
& x_{i}=0 \text { or } 1 \quad i=1,2,3 .
\end{aligned}
$$

From theorem (5.4), if $x^{*}$ is an optimal solution to the weighted problem (6.3) for some $\omega>0$, then $x^{*}$ is an R-efficient solution for (FCP) (6.1). The solution of the problem depends on the choice of the weights in problem (6.3). For example. if the decision maker sets $\omega_{1}=0.5, \omega_{2}=0.25, \omega_{3}=0.25$, then the optimal solution is $\left(x_{1}^{*}, x_{2}^{*}, x_{3}^{*}\right)=(1,1,0)$.

Example 7.2. Consider (FCP) (6.1). Set $k=4$ and

$$
R(\widetilde{A})=\left(R_{1}(\widetilde{A}), R_{2}(\widetilde{A}), R_{3}(\widetilde{A}), R_{4}(\widetilde{A})\right)^{T}
$$

where $R_{1}(\widetilde{A}), R_{2}(\widetilde{A}), R_{3}(\widetilde{A})$ and $R_{4}(\widetilde{A})$ are defined as

$$
\begin{aligned}
& R_{1}(\widetilde{A})=\inf \{x \in R \mid \widetilde{A}(x) \geq 0\} \\
& R_{2}(\widetilde{A})=\inf \{x \in R \mid \widetilde{A}(x) \geq 1\} \\
& R_{3}(\widetilde{A})=\sup \{x \in R \mid \widetilde{A}(x) \geq 1\} \\
& R_{4}(\widetilde{A})=\sup \{x \in R \mid \widetilde{A}(x) \geq 0\}
\end{aligned}
$$

then

$$
\begin{array}{lll}
R_{1}\left(\widetilde{c}_{1}\right)=5, & R_{1}\left(\widetilde{c}_{2}\right)=16, & R_{1}\left(\widetilde{c}_{3}\right)=24 \\
R_{2}\left(\widetilde{c}_{1}\right)=6, & R_{2}\left(\widetilde{c}_{2}\right)=17, & R_{2}\left(\widetilde{c}_{3}\right)=25 \\
R_{3}\left(\widetilde{c}_{1}\right)=7, & R_{3}\left(\widetilde{c}_{2}\right)=18, & R_{4}\left(\widetilde{c}_{3}\right)=26 \\
R_{4}\left(\widetilde{c}_{1}\right)=20, & R_{4}\left(\widetilde{c}_{2}\right)=40, & R_{4}\left(\widetilde{c}_{3}\right)=50
\end{array}
$$

The following (MOLP) is associated with above problem

$$
\begin{aligned}
(\mathrm{MOLP}) \min z(x)= & \left(5 x_{1}+16 x_{2}+24 x_{3}, 6 x_{1}+17 x_{2}+25 x_{3},\right. \\
& \left.7 x_{1}+18 x_{2}+26 x_{3}, 20 x_{1}+40 x_{2}+50 x_{3}\right)^{T}
\end{aligned}
$$

subject to

$$
\begin{aligned}
& x_{1}+x_{2} \geq 1 \\
& x_{2}+x_{3} \geq 1 \\
& x_{1}+x_{3} \geq 1 \\
& x_{i}=0 \text { or } 1 ; \quad i=1,2,3 .
\end{aligned}
$$


Example 7.3. Consider FCP problem (6.1). If we set $k=5$ and

$$
R(\widetilde{A})=\left(R_{1}(\widetilde{A}), R_{2}(\widetilde{A}), R_{3}(\widetilde{A}), R_{4}(\widetilde{A}), R_{5}(\widetilde{A})\right)^{T}
$$

where $R_{1}(\widetilde{A}), R_{2}(\widetilde{A}), R_{3}(\widetilde{A})$ and $R_{4}(\widetilde{A})$ are defined as

$$
\begin{aligned}
& R_{1}(\widetilde{A})=\inf \{x \in R \mid \widetilde{A}(x) \geq 0\} \\
& R_{2}(\widetilde{A})=\inf \{x \in R \mid \widetilde{A}(x) \geq 1\} \\
& R_{3}(\widetilde{A})=\sup \{x \in R \mid \widetilde{A}(x) \geq 1\} \\
& R_{4}(\widetilde{A})=\sup \{x \in R \mid \widetilde{A}(x) \geq 0\} \\
& R_{5}(\widetilde{A})=A(\widetilde{A})
\end{aligned}
$$

then

$$
\begin{array}{lll}
R_{1}\left(\widetilde{c}_{1}\right)=5, & R_{1}\left(\widetilde{c}_{2}\right)=16, & R_{1}\left(\widetilde{c}_{3}\right)=24 \\
R_{2}\left(\widetilde{c}_{1}\right)=6, & R_{2}\left(\widetilde{c}_{2}\right)=17, & R_{2}\left(\widetilde{c}_{3}\right)=25 \\
R_{3}\left(\widetilde{c}_{1}\right)=7, & R_{3}\left(\widetilde{c}_{2}\right)=18, & R_{3}\left(\widetilde{c}_{3}\right)=26 \\
R_{4}\left(\widetilde{c}_{1}\right)=20, & R_{4}\left(\widetilde{c}_{2}\right)=40, & R_{4}\left(\widetilde{c}_{3}\right)=50 \\
R_{5}\left(\widetilde{c}_{1}\right)=17 / 6, & R_{5}\left(\widetilde{c}_{2}\right)=13 / 3, & R_{5}\left(\widetilde{c}_{3}\right)=14 / 3
\end{array}
$$

The following (MLCP) is associated with problem (6.1).

$$
\begin{aligned}
(\mathrm{MLCP}) \min z(x) & =\left(5 x_{1}+16 x_{2}+24 x_{3}, 6 x_{1}+17 x_{2}+25 x_{3}, 7 x_{1}+18 x_{2}\right. \\
& \left.+26 x_{3}, 20 x_{1}+40 x_{2}+50 x_{3}, \frac{17}{6} x_{1}+\frac{13}{3} x_{2}+\frac{14}{3} x_{3}\right)^{T}
\end{aligned}
$$

subject to

$$
\begin{aligned}
& x_{1}+x_{2} \geq 1 \\
& x_{2}+x_{3} \geq 1 \\
& x_{1}+x_{3} \geq 1 \\
& x_{i}=0 \text { or } 1 ; \quad i=1,2,3 .
\end{aligned}
$$

To solve the above problem, the following weighted problem is considered.

$$
\begin{aligned}
\min z(x)=\omega_{1} & \left(5 x_{1}+16 x_{2}+24 x_{3}\right)+\omega_{2}\left(6 x_{1}+17 x_{2}+25 x_{3}\right) \\
& +\omega_{3}\left(7 x_{1}+18 x_{2}+26 x_{3}\right)+\omega_{4}\left(20 x_{1}+40 x_{2}+50 x_{3}\right) \\
& +\omega_{5}\left(\frac{17}{6} x_{1}+\frac{13}{3} x_{2}+\frac{14}{3} x_{3}\right)
\end{aligned}
$$


subject to

$$
\begin{aligned}
& x_{1}+x_{2} \geq 1 \\
& x_{2}+x_{3} \geq 1 \\
& x_{1}+x_{3} \geq 1 \\
& x_{i}=0 \text { or } 1 ; \quad i=1,2,3 .
\end{aligned}
$$

From theorem (5.4), if $x^{*}$ is an optimal solution to the weighted problem (6.6) for some $\omega>0$. then $x^{*}$ is an R-efficient solution for (FCP) (6.1). The solution of the problem depends on the choice of the weights in problem (6.6). For example, if the decision maker sets $\omega_{1}=\omega_{2}=\omega_{3}=\omega_{4}=0.1$ and $\omega_{5}=0.6$, then the optimal solution is $\left(x_{1}^{*}, x_{2}^{*}, x_{3}^{*}\right)=(1,1,0)$.

\section{Conclusion}

In this paper a linear set covering problem with fuzzy parameters in the objective function is being considered.Given a fuzzy linear set covering problem, the multiobjective set covering problem is a formal frame to find solutions for it.In this paper it has been shown that in solving this multiobjective set covering problem by weighting the objectives the solution is obtained.Obviously this is not the only way to solve the multiobjective problem considered and therefore one can assure that according to both the interests of the decision maker and the nature of the problem, different methods of solution may be used to obtain different optimal solutions. The approach for solving this problem uses different ranking functions. To improve the draw back of using a single characteristic,a $\mathrm{k}$-dimensional vector ranking function to a fuzzy number is being associated.

\section{References}

[1] R. Bellman, L.A. Zadeh, Decision making in fuzzy environment, Management Sci. B, 17 (1970), 141-164.

[2] G. Bortolan, R. Degani, A review of some methods for ranking fuzzy numbers, Fuzzy Sets and Systems, 15 (1985), 1-19.

[3] M. Zangiabadi, H.R. Maleki, A method for solving linear programming problems with fuzzy parameters based on multiobjective linear programming technique, Asia Pecific Journal of Operational Research, 24, No. 4 (2007), 557-573. 
[4] R. Steuer, Multiple Criteria Optimization: Theory, Computation and Application, Wiley (1986).

[5] M. Delgado, J.L. Verdegay, M.A. Vila, Imprecise costs in mathematical programming problems, Control and Cybernetics, 16, No. 2 (1987), 113121.

[6] M. Delgado, J.L. Verdegay, M.A. Vila, A general model for fuzzy linear programming, Fuzzy Sets and Systems, 29 (1989), 21-29.

[7] M. Delgado, J.L. Verdegay, M.A. Vila, W. Voxman, A fuzziness measure for fuzzy numbers: Applications, Fuzzy Sets and Systems, 94 (1998), 205216.

[8] J.M. Cadenas, J.L. Verdegay, Using fuzzy numbers in linear programming, IEEE Transactiona on Systems, Man, and Cybernetics (Part B), 27, No. 6 (1997), 1016-1022.

[9] O.L. Mangasarian, Non Linear Programming, McGraw-Hill, New York (1969). 
